Archived version from NCDOCKS Institutional Repository - http://libres.uncg.edu/ir/asu/

Bergman, R. J., Spellman, J. W., Hall, M. E., \& Bergman, S. M. (2012). Is There a Valid App for That? Validity of a Free Pedometer iPhone Application. Journal of Physical Activity and Health, 9(5): 670-676 (July 2012). Published by Human Kinetics (ISSN: 1543-3080). The version of record is available from: www.humankinetics.com

\title{
Is There a Valid App for That? Validity of a Free Pedometer iPhone Application
}

Randall J. Bergman, Justin W. Spellman, Michael E. Hall, and Shawn M. Bergman

\begin{abstract}
This study examined the validity of a selected free pedometer application (iPedometer; IP) for the iPhone that could be used to assess physical activity.

Methods: Twenty college students (10 men, 10 women; mean age: $21.85 \pm 1.57$ yrs) wore an iPhone at 3 locations (pocket, waist, arm) and a StepWatch 3 Step Activity Monitor (SW) on their right ankle while walking on a treadmill at 5 different speeds $(54,67,80,94,107 \mathrm{~m} \cdot \mathrm{min}-1)$. A research assistant counted steps with a tally counter (TC). Results: Statistical significance between the TC, SW, and IP was found during every condition except IP in the pocket at $107 \mathrm{~m} \cdot \mathrm{min}-1(F 2,38=.64, P=.54)$. Correlations involving the IP revealed only 1 positive correlation (IP on arm at 54 $\mathrm{m} \cdot \mathrm{min}-1)$ for any of the conditions $(r=.46, P=.05)$. Conclusion: The IP application was not accurate in counting steps and recorded significantly lower step counts than the SW and TC. Thus, the free pedometer application used is not a valid instrument for monitoring activity during treadmill walking.
\end{abstract}


Although the benefits of physical activity are widely known and well documented ${ }^{1-3}$ many people are insufficiently active to achieve health benefits. This lack of physical activity is one component of the obesity epidemic in the United States. Healthy People 2020 has objectives to increase the amount of physical activity performed by all Americans. ${ }^{4}$ All Americans are encouraged to accumulate 150 minutes of mod- erate-intensity activity per week to promote cardio- respiratory health. ${ }^{2,5}$ Studies have shown that 30 minutes of brisk walking is equal to 3100 to 4000 steps, depending on age.${ }^{6,7}$ Researchers advocate taking a total of 10,000 steps per day for cardiovascular disease prevention. ${ }^{8}$

The emphasis on increasing physical activity has driven the need to accurately assess the amount of activity an individual participates in per day. Pedometers and accelerometers can motivate individuals to be more physically active and have become the standard tool for objectively measuring physical activity. ${ }^{7,9-11}$ Studies have reported the accuracy of different pedometers in assessing physical activity at different walking speeds. ${ }^{12-15}$ In general, it has been shown that waist-mounted pedometers increase in accuracy as walking speed increases. ${ }^{12}$

Since the iPhone 3G premiered in the U.S. in July of 2008 it has increased in popularity, currently it ranks second in U.S. cellular phone sales. ${ }^{16}$ Typically, cellular phones do not have specific placement requirements for the pedometer applications because of the built-in accelerometer. ${ }^{17}$ Their placement depends on where the user is most comfortable carrying the phone. It could be placed in a pocket, attached to the waist band with a clip or to the arm with an armband. Other pedometers have placement requirements (midline of the thigh) due to the mechanisms used for recording steps. The location of these pedometers may affect their accuracy. ${ }^{18}$ When considering the recommendations for physical activity and the popularity of the iPhone and its many applications, it is important to have these pedometer applications validated. The purpose of this study was to investigate the accuracy of one of the free pedometer applications for the iPhone.

\section{Methods Participants}

A convenience sample of 20 healthy college students [ 10 men, 10 women; mean age $=21.85 \pm 1.57$; body mass index $(B M I)=26.24 \pm 5.80]$ were recruited for this study. The sole exclusion criterion was being nonambulatory. The procedures were approved by the Institutional Review Board (IRB) at Missouri Western State University. Each participant completed a Physical Activity Readiness Questionnaire (PAR-Q) and signed an informed consent before participating. Physical characteristics of the participants are presented in Table 1. 
Table 1 Physical Characteristics of the Participants

\begin{tabular}{lccc}
\hline & Men $(\mathbf{N}=10)$ & Women $(\mathbf{N}=10)$ & All particlpants $(\mathbf{N}=\mathbf{2 0})$ \\
\hline Age $(\mathrm{yrs})$ & $21.90 \pm 1.85$ & $21.80 \pm 1.31$ & $21.85 \pm 1.57$ \\
Height $(\mathrm{cm})$ & $180.77 \pm 10.98$ & $168.70 \pm 0.04$ & $174.73 \pm 10.15$ \\
Weight $(\mathrm{kg})$ & $92.96 \pm 15.18$ & $68.14 \pm 14.79$ & $80.55 \pm 19.36$ \\
BMI $\left(\mathrm{kg} \cdot \mathrm{m}^{-2}\right)$ & $28.68 \pm 6.15$ & $23.79 \pm 4.45$ & $26.24 \pm 5.80$ \\
\hline
\end{tabular}

Note. Values are means \pm standard deviation.

Abbreviations: BMI, Body Mass Index.

\section{Protocol}

Procedures were similar to those used by Crouter, Schneider, Karabulut, and Bassett. ${ }^{12}$ All participants wore athletic shorts with pockets, comfortable walking shoes, and had their height and weight measured with- out shoes with a stadiometer and calibrated physician's scale. For the first trial the participant placed the iPhone (Apple, Inc.) in their right pocket. The second trial the phone was secured to the waist band at the midline of the right thigh using a horizontal case. In the third trial it was secured to the upper right arm using an armband. The walking speeds for each trial were 54, 67, 80, 94, $107 \mathrm{~m} \cdot \mathrm{min}^{-1}(2.0,2.5,3.0,3.5$, $4.0 \mathrm{mph}$ ) at $0 \%$ grade on a motor driven treadmill (Quinton Instrument Company). An ankle-mounted StepWatch 3 Step Activity Monitor (SW; Orthocare Innovations; $r>=.99 ; 19-20$ ) was placed on the right ankle and set to normal sensitivity. A research assistant counted steps with a tally counter (TC). The participant would walk at the given speed for 2 minutes then stop and straddle the belt for 2 minutes. While straddling the belt values of the iPhone pedometer application and TC were recorded onto a data collection sheet and the treadmill speed increased. This break also allowed the data collected from the SW to be easily separated after downloading it into the computer software at the end of all trials. A total of 5 SWs and 5 IPs were rotated. 


\section{Instruments}

All of the phones used in this study were $3 \mathrm{G}, 16 \mathrm{G}$ with built-in accelerometers. Version 1 (released in September of 2009) of the free pedometer application, iPedometer (Tomato, Inc.), (IP) was downloaded to each phone. The SW pedometers used in this study are completely sealed microprocessor-controlled step counters. Programming and downloading are controlled with the StepWatch Analysis Software. This software programs the SW monitor before deployment, and downloads it to the computer via a USB-compatible docking station for viewing at the end of the recording session. Sensitivity of the instrument is optimized for each subject's gait characteristics by programming in the subject's height and answering questions that describe the subject's gait. Data were collected in 1-minute time intervals.

\section{Statistical Analysis}

All analyses were conducted using SPSS 17.0 for Windows (SPSS Inc., Chicago, IL). Statistical significance was set at $P<.05$ unless otherwise noted. The SW and TC only counted steps for 1 leg, therefore SW and TC steps were doubled to enable comparison.

One-way repeated-measures ANOVAs were used to assess differences between pedometers and observed step counts. In the case of a significant main effect, Least Significant Difference (LSD) post hoc analysis was used to determine between which methods of measurement differences occurred. Correlations between pedometers and observed step counts were examined. Data were analyzed using Pearson product moment correlations for the IP, SW, and TC.

Error scores were computed (TC minus pedometers steps) to determine congruency, according to the method of Bland and Altman. ${ }^{21}$ This graphical representation shows the variability in individual step counts around 0 and signifies the best possible estimate of the actual value. The mean error score can be illustrated and the $95 \%$ confidence interval (for individual observations) can also be denoted. Tight prediction intervals around 0 signify that measures for the 2 comparison devices are congruent. Scores over 0 (positive scores) indicate underestimation of steps relative to the TC and scores under 0 (negative scores) indicate overestimation of steps relative to the TC. 


\section{Results}

Data analysis indicated statistically significant differences $(P<.001)$ for every iPhone location and speed except for in the pocket at $107 \mathrm{~m} \cdot \mathrm{min}^{-1}\left(F_{2,38}=0.64, P\right.$ $=.54)$. Post hoc analysis with a LSD adjustment revealed that all differences occurred between the IP and SW $(P<.001)$ and the IP and TC $(P<.001)$. For all analyses the observed power was greater than 0.99 , except for $107 \mathrm{~m} \cdot \mathrm{min}^{-1}$ with the iPhone in the pocket which had a power of $0.15 .^{22}$ Table 2 illustrates the mean error scores, standard error, and 95\% confidence intervals between the TC and the pedometers' measured steps for each condition. Figures 1 to 3 show Bland-Altman plots for selected conditions for each pedometer to illustrate the distribution of the individual error scores around 0 and indicate the best possible guess of the true value being measured. The IP significantly underestimated the observed number of steps taken as recorded by the TC.

Pearson product moment correlations $(r)$ involving the IP revealed only 1 positive correlation (iPhone on arm at $54 \mathrm{~m} \cdot \mathrm{min}^{-1} ; r=.46, P=.05$; Figure 4) for any of the conditions. All correlations between the SW and TC were highly correlated $(r$ $.97, P<.001)$. Three selected conditions are shown as scatter plots in Figures 5 to 7. 
Table 2 Error Scores (TC-Pedometers) in Number of Steps for All Conditions

\begin{tabular}{|c|c|c|c|c|c|}
\hline Location & Pedometer & Speed $\left(\left.m \cdot m\right|^{-1}\right)$ & Mean difference & SE & $95 \%$ confidence Interval \\
\hline \multirow[t]{10}{*}{ Pocket } & SW & 54 & -.70 & .53 & -2.09 to .69 \\
\hline & IP & & $100.15^{* *}$ & 10.14 & 73.53 to 126.77 \\
\hline & & 67 & -.30 & .54 & -1.74 to 1.14 \\
\hline & & & $95.30^{* *}$ & 12.61 & 62.21 to 128.39 \\
\hline & & 80 & -.60 & .36 & -1.54 to .34 \\
\hline & & & $57.30^{* *}$ & 16.00 & 15.29 to 99.31 \\
\hline & & 94 & -.40 & .34 & -1.30 to .50 \\
\hline & & & $31.50^{*}$ & 15.42 & -9.97 to 70.97 \\
\hline & & 107 & -.40 & .45 & -1.58 to .78 \\
\hline & & & -15.85 & 19.72 & -67.63 to 35.93 \\
\hline \multirow[t]{10}{*}{ Waist } & sw & 54 & -.30 & .33 & -1.18 to .58 \\
\hline & IP & & $164.95^{* *}$ & 5.46 & 150.62 to 179.28 \\
\hline & & 67 & -.20 & .38 & -1.20 to .80 \\
\hline & & & $176.75^{* *}$ & 5.90 & 161.27 to 192.23 \\
\hline & & 80 & -.40 & .37 & -1.38 to .58 \\
\hline & & & $176.20 * *$ & 7.64 & 156.14 to 196.26 \\
\hline & & 94 & -.80 & .40 & -1.84 to .24 \\
\hline & & & $163.20 * *$ & 9.63 & 137.91 to 188.49 \\
\hline & & 107 & -.30 & .49 & -1.58 to .98 \\
\hline & & & $116.75^{* *}$ & 13.83 & 80.46 to 153.04 \\
\hline \multirow[t]{10}{*}{ Arm } & SW & 54 & .50 & .56 & -.97 to 1.97 \\
\hline & IP & & $186.30 * *$ & 2.43 & 180.41 to 193.19 \\
\hline & & 67 & .40 & .57 & -1.10 to 1.90 \\
\hline & & & $207.60^{* *}$ & 2.50 & 201.05 to 214.15 \\
\hline & & 80 & -.50 & .48 & -1.76 to .76 \\
\hline & & & $219.75^{* *}$ & 2.79 & 212.43 to 227.07 \\
\hline & & 94 & -.60 & .60 & -2.18 to .98 \\
\hline & & & $229.40 * *$ & 3.50 & 220.20 to 238.60 \\
\hline & & 107 & -1.30 & .69 & -3.01 to .50 \\
\hline & & & $224.60 * *$ & 6.56 & 207.39 to 241.81 \\
\hline
\end{tabular}

Note. Negative scores indicate overestimations and positive scores correspond with underestimations of observed steps taken.

$* P<.05 ; * * P<.001$.

Abbreviations: TC, Tally Counter; SW, StepWatch; IP, iPedometer. 

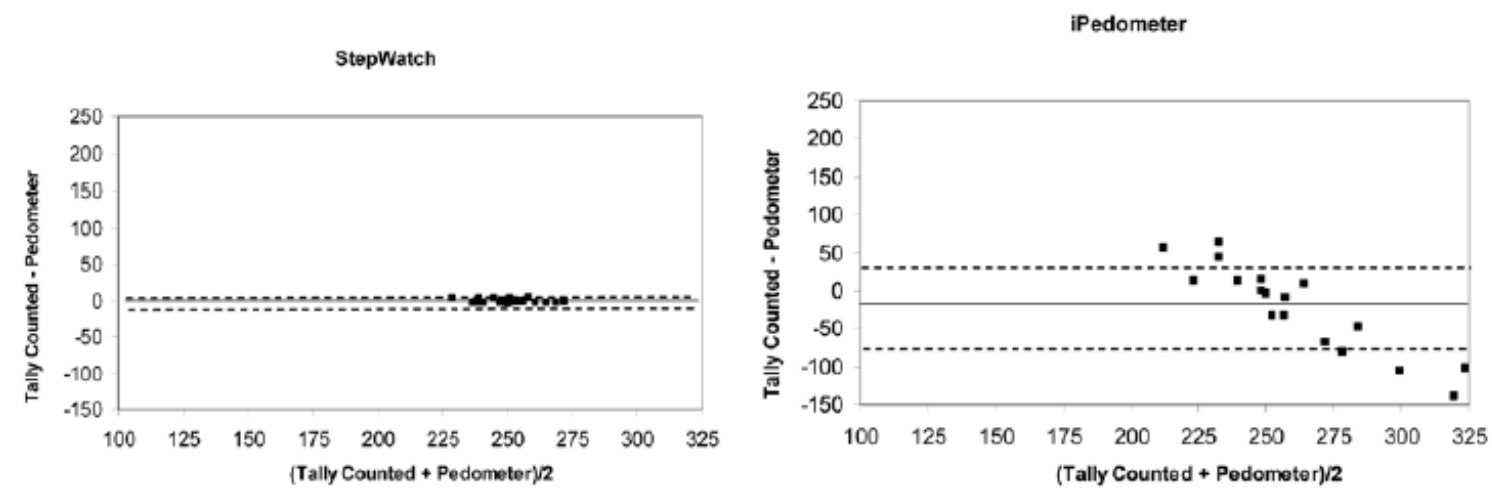

Figure 1 - Representative Bland-Altman plots for pedometers at $107 \mathrm{~m} \cdot \mathrm{min}^{-1}$ and iPhone in pocket. Solid line is mean error score; dashed lines are $95 \%$ confidence intervals.
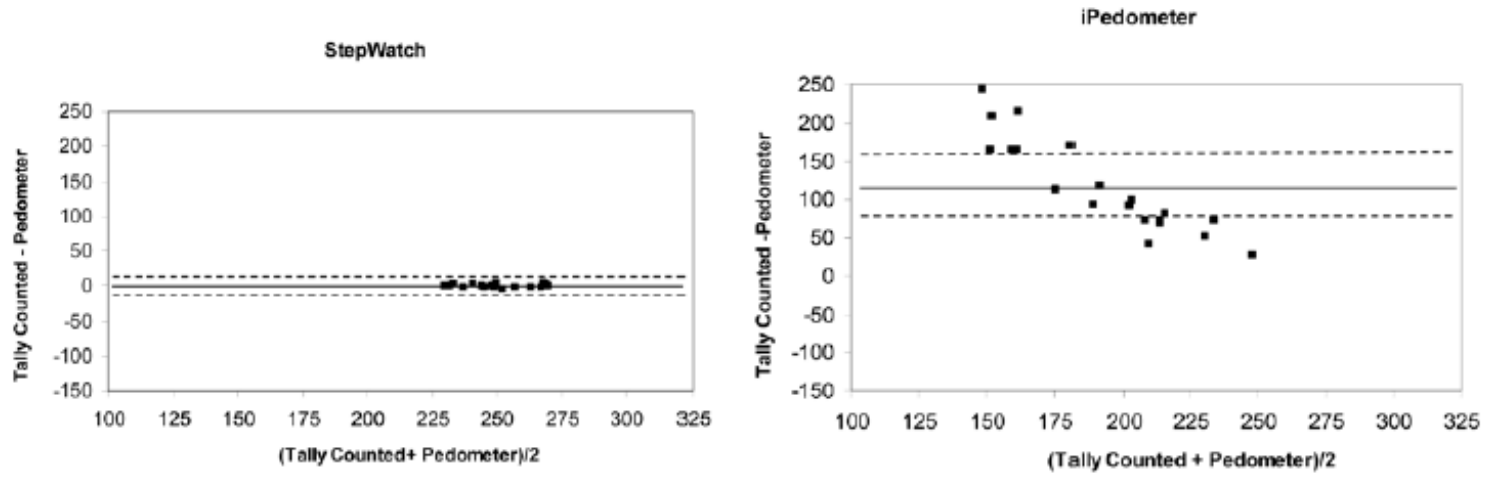

Figure 2 - Representative Bland-Altman plots for pedometers at $107 \mathrm{~m} \cdot \mathrm{min}^{-1}$ and iPhone on waist. Solid line is mean error score; dashed lines are $95 \%$ confidence intervals.

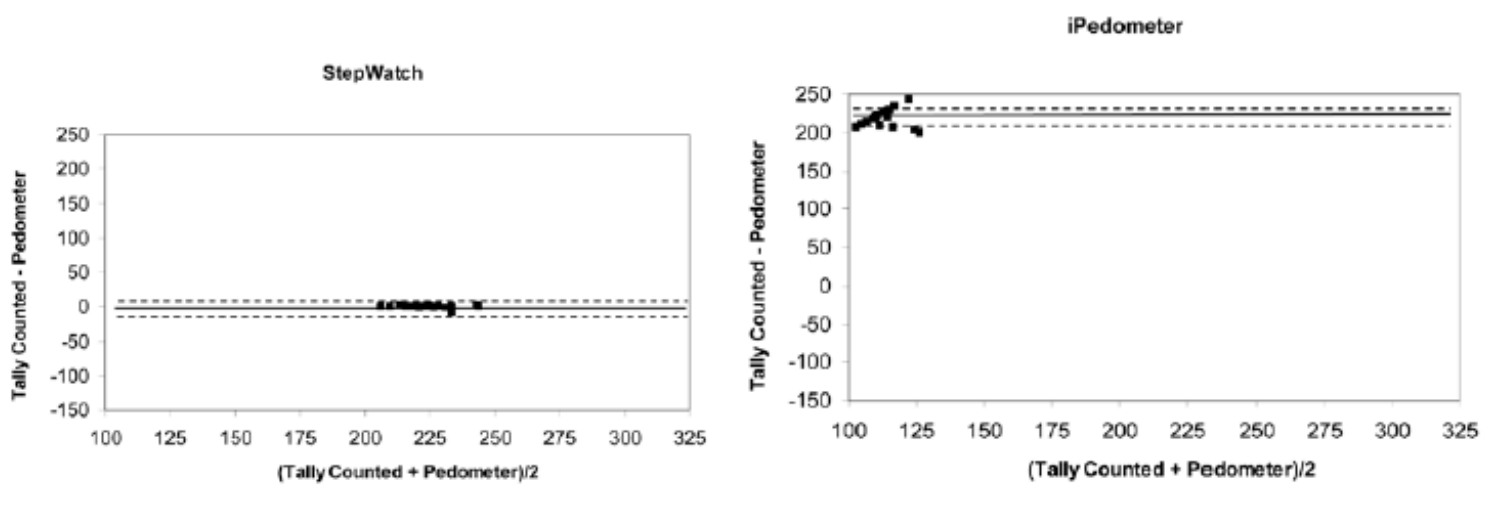

Figure 3-Representative Bland-Altman plots for pedometers at $80 \mathrm{~m} \cdot \mathrm{min}^{-1}$ and iPhone on arm. Solid line is mean error score; dashed lines are $95 \%$ confidence intervals. 


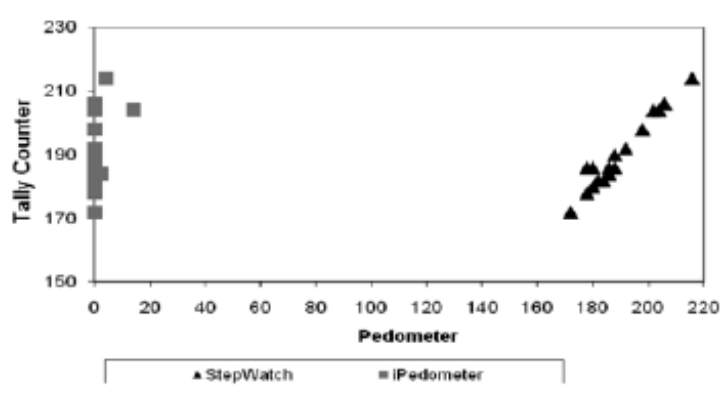

Figure 4 - Scatter plot of steps tally counted vs. pedometers with speed at $54 \mathrm{~m} \cdot \mathrm{min}^{-1}$ and iPhone on the arm. StepWatch positively correlated $(r=.98, P<.001)$; iPedometer positively correlated $(r=.46, P=.05)$.

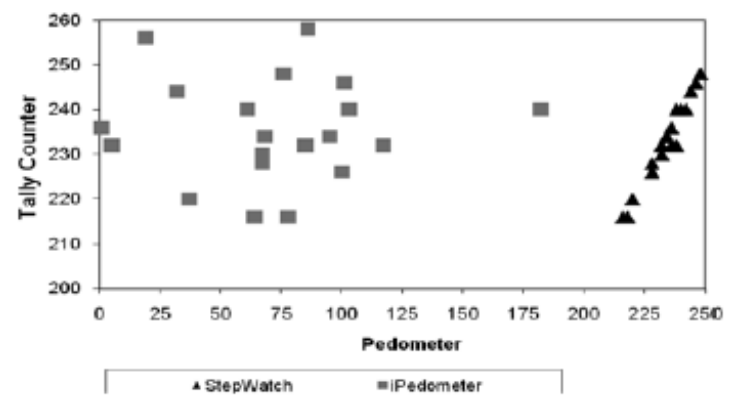

Figure 6 - Scatter plot of steps tally counted vs. pedometers with speed at $94 \mathrm{~m} \cdot \mathrm{min}^{-1}$ and iPhone on waist. StepWatch positively correlated $(r=.99, P<.001)$; iPedometer not correlated $(r=-0.08, P=.73)$.

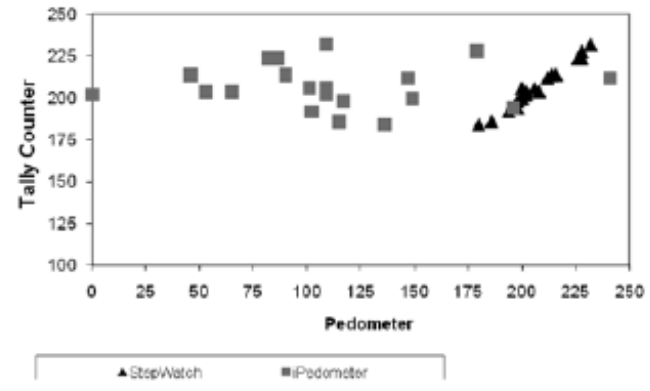

Figure 5-Scatter plot of steps tally counted vs. pedometers with speed at $67 \mathrm{~m} \cdot \mathrm{min}^{-1}$ and iPhone in pocket. StepWatch positively correlated $(r=.99, P<.001)$; iPedometer not correlated $(r=-0.03, P=.91)$.

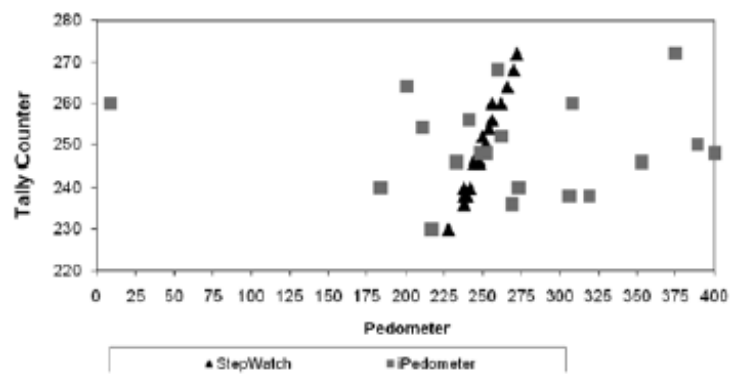

Figure 7 - Scatter plot of steps tally counted vs. pedometers with speed at $107 \mathrm{~m} \cdot \mathrm{min}^{-1}$ and iPhone in pocket. StepWatch positively correlated $(r=.99, P<.001)$; iPedometer not correlated $(r=-0.05, P=.82)$. 


\section{Discussion}

This study found that the IP application for the iPhone yielded mean values that were significantly lower than the observed and SW means. The location of the IP (pocket, waist, arm) or walking speeds $\left(54,67,80,94,107 \mathrm{~m} \cdot \mathrm{min}^{-1}\right)$ did not increase the accuracy of the pedometer application. Although data analysis revealed no statistically significant difference with the IP in the pocket at 107 $\mathrm{m} \cdot \mathrm{min}^{-1}$, Pearson product moment correlations did not illustrate a significant correlation for that location and speed.

At these placement locations and walking speeds the IP was not accurate in step counting. This result is possibly due to the acceleration of the movement not producing enough force for a step to be detected. The accelerometer used in the iPhone is a micro electro- mechanical system (MEMS) $\cdot{ }^{17}$ that consist of a deflection circuit and piezo-electric crystal with a cantilever beam. ${ }^{23}$ With most healthy adults walking at approximately $84 \mathrm{~m} \cdot \mathrm{min}^{-1},{ }^{24}$ the last 3 speeds used in this study should produce enough force to register a step according to other pedometer studies. ${ }^{12,15,19}$ This could be due to the location of the IP during the trials. These 3 locations were selected as typical use but other locations could be back pocket, hand bag or backpack.

If there was indeed a fundamental design flaw with the accelerometer of the iPhone that could not be addressed with a new algorithm, no pedometer application would be accurate. A second possible reason for the inaccuracy of the IP is that the calculations within the IP software itself are incorrect. Calculation errors within the software can be resolved by implementing a better algorithm for the IP via software patch or selection of a more accurate pedometer application. Finally, it is possible that the IP or the iPhone cannot sample at a fast enough rate to count all of the steps. Like previously stated, if the sampling issue is with the application software that is easier to resolve. The software can be improved to record at a faster rate or another application can be downloaded. If the problem is with the internal accelerometer then no other application could be expected to accurately register steps. There are numerous free and paid for pedometer applications available that could have more accurate algorithms and quicker sampling rates that would greatly enhance the ability to precisely count steps. This study supports previous research that the SW is a valid step counter ${ }^{19,20,25-29}$ with $100 \%$ step detection.

Our study is not without limitations. It is acknowledged that treadmill walking is not representative of normal walking. However, it is valuable to assess step counters at known walking speeds. It is also recognized that both the IP and SW are expensive ( $\$ 300$ plus service, $\$ 500$ plus $\$ 1500$ docking station and software, respectively) and might not be the best choice for measuring activity in the general 
population. The small convenience sample limits the generalizability of this study. The sample was chosen due to the large number of college students who use the iPhone. Similar studies used convenient samples comparable in size ${ }^{12,15,19,20}$ and the reported statistical power is strong enough to show effect without having to increase sample size. In addition, there was no attempt to test the reliability of the TC. Future research should consider video recording each trail to ensure accuracy of the manually counted steps.

In conclusion, the current study found that the free iPedometer application for the iPhone is not a valid instrument when measuring activity at selected placements and walking speeds. The current study only tested one of the numerous free pedometer applications available for the iPhone. Further research with the other pedometer applications, both free and paid, are needed to assess their accuracy at different placements and speeds. In addition, future research should consider using other statistical techniques, such as equivalency testing; to examine the accuracy of pedometers. With the increasing number of adults in the U.S. becoming overweight meeting the recommendations for physical activity is important. Since walking is the primary form of activity for the general population, and with the popularity of "all-in-one" electronic devices, it is essential that pedometer applications on these devices be able to accurately measure steps. 


\section{References}

1. Mazzeo R, Cavanaugh P, Evans W, et al. ACSM position stand on exercise and physical activity for older adults. Med Sci Sports Exerc. 1998;30(6):9921008. PubMed doi:10.1097/00005768-199806000-00033

2. U.S. Department of Health and Human Services. 2008 Physical activity guidelines for Americans: Be active, healthy, and happy. Retrieved August, 2010 from: www. health/gov/paguidelines

3. Center for Disease Control and Prevention. Physical activity. Retrieved August, 2010 from: www.cdc.gov/ physicalactivity/index.html.

4. Healthy People 2020. Healthy People 2020. Retrieved August, 2010 from www.healthypeople.gov/, 2009.

5. HaskellW,LeeR,PateR,etal.Physicalactivityandpublic health: updated recommendation for adults from the American College of Sports Medicine and the American Heart Association. Med Sci Sports Exerc. 2007;39(8):14231434. PubMed doi:10.1249/mss.0b013e3180616b27

6. Tudor-LockeC,JonesR,MyersD,PatersonH,Ecclestone N. Contribution of structured exercise class participation and informal walking for exercise to daily physical activity in community-dwelling older adults. Res $Q$ Exerc Sport. 2002;73:350-356. PubMed

7. Welk G, Differding R, Thompson S, Blair N, Dziura J, Hart P. The utility of the Digi-Walker step counter to assess daily physical activity patterns. Medicine and Science in Exercise and Sport. 2002;32:S481-S488. PubMed doi:10.1097/00005768-200009001-00007

8. Hatano Y. Prevalence and use of the pedometer. Research Journal in Walking. 1997;1:45-54.

9. Sequeira M, Rickenbach M, Wietlisbach V, Tullen B, Schutz Y. Physical activity assessment using a pedometer and its comparison with a questionnaire in a large population survey. Am J Epidemiol. 1995;142:989-999. PubMed

10.10. Speck B, Looney S. Effects of a minimal intervention to increase physical activity in women: daily activity records. Nurs Res. 2001;50:374-378. PubMed doi:10.1097/00006199-200111000-00008 
11. Bravata D. Using pedometers to increase physical activity and improve health. JAMA. 2007;298(19):2296. PubMed doi:10.1001/jama.298.19.2296

12. Crouter S, Schneider P, Karabulut M, Bassett D. Validity of 10 electronic pedometers for measuring steps, distance, and energy cost. Med Sci Sports Exerc. 2003;35(8):14551460. PubMed doi:10.1249/01.MSS.0000078932.61440. A2

13. Schneider P, Crouter S, Lukajic O, Bassett D. Accuracy and reliability of 10 pedometers for measuring steps over a 400-m walk. Med Sci Sports Exerc. 2003;35(10):1779- 1784. PubMed doi:10.1249/01.MSS.0000089342.96098. C4

14. BassettD,AinsworthB,LeggettS, etal.Accuracyoffive electronic pedometers for measuring distance walked. Med Sci Sports Exerc. 1996;28(8):1071-1077. PubMed doi:10.1097/00005768-199608000-00019

15. Schneider P, Crouter S, Bassett D. Pedometer measures of free-living physical activity: comparison of 13 models. Medicine and Science in Exercise and Sport. 2004;36(2):331-335. PubMed doi:10.1249/01. MSS.0000113486.60548.E9

16. Bloomberg Businessweek. Top-selling U.S. cell phones. Retrieved August, 2010 from: www.businessweek.com/ images/ss/09/02/0204_cellphones/index.htm.

17. ChenC,AntonS,AbdelsalamH.Abriefsurveyofphysical activity monitory devices. Mobile \& Pervasive Computing Research, University of Florida. May 2008.

18. Oliver M, Schofield G, Kolt G, Schluter P. Pedometer accuracy in physical activity assessment of preschool children. J Sci Med Sport. 2007;10:303-310. PubMed doi:10.1016/j.jsams.2006.07.004

19. Karabulut M, Crouter S, Bassett D. Comparison of two waist-mounted and two anklemounted electronic pedometers. Eur J Appl Physiol. 2005;95:335-343. PubMed doi:10.1007/s00421-005-0018-3

20. Foster R, Lanningham-Foster L, Manohar C, et al. Precision and accuracy of an ankle-worn accelerometer-based pedometer in step counting and energy expenditure. Prev Med. 2005;41:778-783. PubMed doi:10.1016/j. ypmed.2005.07.006

21. Bland J, Altman D. Statistical methods for assessing agreement between two methods of clinical measurement. Lancet. 1986;1:301-310. PubMed

22. FaulF,ErdfelderE,LangAG,Buchner,A.G*Power3:A flexible statistical power analysis program for the social, behavioral, and biomedical sciences. Behav Res Methods. 2007;39:175-191. PubMed doi:10.3758/BF03193146

23. An introduction to accelerometers: using sensors to measure acceleration. Retrieved August, 2010 from: engineering.suite101.com/article.cfm/an_introduction_to_ accelerometers. 
24. Temes W. Cardiac rehabilitation. In: Hillegass E, Sadowsky H, eds. Essentials of cardiopulmonary physical therapy. Philadelphia: W.B. Saunders; 1994:633-676.

25. Macko R, Haeuber E, Shaughnessy M, et al. Microprocessor-based ambulatory activity monitoring in stroke patients. Med Sci Sports Exerc. 2002;34:394-399. PubMed doi:10.1097/00005768-200203000-00002

26. Haeuber E, Shaughnessy M, Forrester L, Coleman K, Macko R. Accelerometer monitoring of home- and com- munity- based ambulatory activity after stroke. Arch Phys Med Rehabil. 2004;85:1997-2001. PubMed doi:10.1016/j. apmr.2003.11.035

27. Coleman K, Smith D, Boone D, Joseph A, del Aguila M. Step activity monitor: longterm, continuous recording of ambulatory function. J Rehabil Res Dev. 1999;36(1):8-18. PubMed

28. Resnick B, Nahm E, Orwig D, Zimmerman S, Magaziner J. Measurement of activity in older adults: reliability and validity of the step activity monitor. J Nurs Meas. 2001;9(3):275-290. PubMed

29. Shaughnessy M, Michael K, Sorkin J, Macko R. Steps after stroke, capturing ambulatory recovery. Stroke. 2005;36:1305-1307. PubMed doi:10.1161/01. STR.0000166202.00669.d2 